\title{
SOMATIC BRCA MUTATION IN CHOLANGIOCARCINOMA PATIENT UTILIZED TO INDIVIDUALIZE AN HBOC SYNDROME
}

\author{
Angelo Paradiso ${ }^{1,2}$, Margherita Patruno ${ }^{1}$, Maria Digennaro ${ }^{1}$, Stefania Tommasi ${ }^{3}$, Brunella Pilato ${ }^{3}$, \\ Antonella Argentiero ${ }^{4}$, Oronzo Brunetti ${ }^{4 \#}$ and Nicola Silvestris ${ }^{4,5 * \#}$ \\ ${ }^{1}$ Experimental Oncology - Center for Study of Heredo-Familial Tumors, IRCCS-Istituto Tumori "Giovanni \\ Paolo II", viale Orazio Flacco, 65, 70124, Bari, Italy. a.paradiso@oncologico.bari.it. \\ ${ }^{2}$ Scientific Direction, Istituto Tumori G Paolo II IRCCS National Cancer Research Centre, Via O Flacco, 65, \\ 70124, Bari, Italy. a.paradiso@oncologico.bari.it. \\ ${ }^{3}$ Molecular and Pharmacogenetics Diagnostic Laboratory, IRCCS-Istituto Tumori "Giovanni Paolo II", viale \\ Orazio Flacco, 65, 70124, Bari, Italy. \\ ${ }^{4}$ Medical Oncology Unit, National Cancer Research Centre, IRCCS Istituto Tumori "Giovanni Paolo II", \\ 70124 Bari, Italy. \\ 5 Department of Biomedical Sciences and Human Oncology, University of Bari "Aldo Moro", 70124 Bari, \\ Italy.
}

\# coolast Authors

* corresponding Author

Medical Oncology Unit, National

Cancer Research Centre, IRCCS Istituto Tumori "Giovanni Paolo II"

Viale O. Flacco 65

70124 Bari, Italy.

Department of Biomedical Sciences and Human Oncology

University of Bari "Aldo Moro"

70124 Bari, Italy.

n.silvestris@oncologico.bari.it. 


\begin{abstract}
$B R C A$-associated hereditary breast and ovarian cancer syndrome (HBOC) implies increased absolute risk also for other malignancies such as cholangiocarcinoma (CCA). However, even if somatic mutations in CCA have been reported, there are no data on its utilization as predisposing genetic factor in a family. For the first time, we utilized CCA somatic BRCA mutation to individualize a family with HBOC. A 47-year-old woman (daughter of a patient died for CCA) accessed to our Genetic Councelling to evaluate her personal cancer risk. We performed a somatic BRCA1/2 NGS analysis on DNA extracted from formalin-fixed, paraffinembedded CCA-tissue of her mother. After demonstration of pathogenic variant c.6468_6469delTC in BRCA2 gene mutation, the same germline pathogenic variant was found in DNA blood of one of two daugthers. So far, CCA tissue can be utilized, in addition to patient's selection to specific therapeutic strategies, also to individualize families belonging to HBOC syndrome.
\end{abstract}

Keywords: BRCA1; BRCA2; biliary tract cancers; ereditary BRCA cancer; somatic mutations 


\section{Introduction}

BReast Cancer (BRCA)1- and BRCA2-associated hereditary breast and ovarian cancer syndrome (HBOC) is characterized by an increased risk for female and male breast cancer, ovarian cancer (includes fallopian tube and primary peritoneal cancers), and to a lesser extent other cancers (prostate cancer, pancreatic cancer, and melanoma) [1-2]. The association of germline mutations in BRCA1/2 genes to the development of bile duct malignancies has previously been reported [3]. Data from the Breast Cancer Linkage Consortium reported that the relative risk of developing gall bladder or bile duct cancer among BRCA2 carriers is 4.97 [3]. The Netherlands Collaborative Group on Hereditary Breast Cancer reported statistically increased relative risks for cancers of the gallbladder and other bile ducts, with relative risks of 3.5 and 5.0, respectively [4]. However, in spite of these evidences, the NCCN guidelines do not consider these tumors among the clinical criteria for access to BRCA1/2 genetic testing in cholangiocarcinoma (CCA) [5]. In addition, utilization of BRCA somatic information to select patients to candidate to specific biological approaches is still not a routinary approach [4]. This situation led to less interest for BRCA status in CCA patients with the scarce studies focused on somatic DNA alterations exclusively [6-7].

For the first time, we report that CCA can be the index case in a family with hereditary ovarian-breast cancer syndrome and, even more important, that somatic CCA DNA test can be informative for risk purposes.

Herein, we report BRCA characterization of a family upon the request of oncogenetic counseling by a 47year-old, healthy woman (daughter of a patient who died for a CCA) to evaluate her personal cancer risk. Since her mother was dead with impossibility to perform a germinal BRCA evaluation, we performed a somatic BRCA1/2 NGS analysis on DNA extracted from formalin fixed, paraffin-embedded CCA biopsy.

\section{Experimental section}

Clinical features of this patient were collected at the National Cancer Institute "Giovanni Paolo II" of Bari, Bari, Italy. All the subjects participating to the study signed the informed consent for this study approved by Ethical Committee (700/18) of National Cancer Institute "Giovanni Paolo II" of Bari, Bari, Italy. The Centro Studi Tumori Eredo-familiari of the Institute managed the genetic counseling of people participating to the study. All the laboratory test were performed in the Molecular Diagnostic Laboratory of the Institute.

DNA was extracted from the whole peripheral blood of each affected proband using the Wizard Genomic DNA Purification kit (Promega Inc., Madison, WI) according to the manufacture's protocol. DNAs were screened for mutations in the entire coding region of the BRCA1 gene. Each BRCA1 coding exon was amplified using intronic primers as previously described [8].

PCR amplification was carried out in a total volume of $50 \mu \mathrm{l}$ containing $100 \mathrm{ng}$ of genomic DNA as a template, $1 \mathrm{X}$ reaction buffer, 1.5-3 mM magnesium chloride, $200 \mu \mathrm{MdNTP}, 20-50 \mathrm{pm}$ each PCR primer and $0.25 \mathrm{U}$ AmpliTaq Gold. After PCR amplifications, $5 \mu \mathrm{l}$ of the reaction product was analyzed via gel electrophoresis and ethidium bromide staining.

DNA sequencing was performed on both strands of two independent PCR products by cycle sequencing on a ABI PRISM 377 automated cycle sequencer, using the Terminator Cycle Sequencing Kit v.1.1 according to the manufacturer's protocol (Applied Biosystems, CA). 
If a mutation was identified, a new DNA aliquot from the same patient was sequenced to confirm the result.

\section{Results}

A 47-year-old, healthy woman, with a family history of cancer, accessed to our Genetic Councelling with the aim to evaluate her personal cancer risk. Her mother at age of 60 s showed multiple liver lesions during preoperative study for uterine prolapse. Liver biopsy diagnosed with hepatic localization of moderately differentiated adenocarcinoma with desmoplastic stroma. The morphological and immunohistochemical profile oriented for a CCA (CK ++; CK19 ++; CEA ++; EMA +++; CAM $5.2+++$; TTF1, CDX2, CK20, ER and PgR: negative).

The proband's maternal grandfather had been diagnosed with skin cancer at age of 80 years and died of a stroke while apparently cancer free 13 years later. A maternal uncle was diagnosed with skin cancer when he was 56 years old. No case of cancer has been reported in the paternal branch (Fig. 1). In the suspicion of a HBOC in her mother, we discussed with the proband and her sister the possibility of performing the genetic test on the pathological material of her mother, now deceased.

After signing of informed consent, we performed BRCA1/2 NGS analysis on DNA extracted from formalinfixed, paraffin-embedded CCA biopsy. The only pathogenic variant identified was c.6468_6469delTC in BRCA2 gene. In the possibility that the identified variant was germinal, we looked for the same variant on the DNA extracted from the lymphocytes of the peripheral blood (PBLs) of her sister. The foundvariant was confirmed in her sister (Fig. 1).

\section{Discussion}

Somatic mutations of BRCA1 and BRCA2 genes have been reported, also, in CCA [9-10]. In spite of this information, biliary tract cancer nowday is not routinely candidate for BRCA1/2 somatic testing.

In clinical practices, mutation of BRCA is a germline evaluation on blood. Overall, the relatives of a patient with BRCA-mutated malignancies require genetic counseling, with the aim of assessing the risk of developing BRCA-addicted neoplasms for entering in specific prevention programs. The assessment of germline BRCA on blood is not always possible (patient dead, tissue not available, patient not senting, etc).

This was the emblematic case of this report. We conducted a BRCA somatic evaluation status on paraffinembedded CCA biopsy of the dead patient. The unique pathogenic variant identified was c.6468_6469delTC in BRCA2 gene. After, we looked for the same variant on the DNA extracted from the PBLs of our proband and her sister. The same mutational variant was confirmed in proband sister. For the first time it was used a CCA somatic BRCA assessment in order to find a HBOC, so far, a genetic test on DNA from a CCA biopsy is performed to assess the presence of a neoplastic predisposition syndrome.

Actually, CCA is a challenging disease in terms of both diagnosis and treatment because of its poor survival and chemoresistant [11]. New therapeutic strategies are warranted. Better biological characterization of this tumor might allow application of targeted therapy for CCA patients with germline or somatic mutations in BRCA1/2 genes. Inhibition of PARP lead to the synthetic lethality in malignancies with BRCA1/2. In a recent retrospective analysis, of 18 patients with BRCA1/2 mutated CCA (6 and 12 patients with extrahepatic and intrahepatic CCA, respectively), four patients underwent to PARP-i, with one of them 
achieving a sustained disease response and a 42.6 months of progression-free survival [12]. Median overall survivals were 40.3 months (95\% confidence interval [CI], 6.73-108.15) and 25 months (95\% CI, 15.2340.57) for stage I/II and stages III/IV patients, respectively. In our report, the availability of the only CCA tissue in a family suspected for HBOC syndrome, forced us, to perform BRCA1/2 genetic analysis on tumor tissue in order to assess personal cancer risk.

Thanks to the NGS sequencing technology, today it is possible to identify in an extremely sensitive way the presence of somatic mutations even from small amounts of tumor tissue. Therefore, where it is not possible to test the affected patient, why not analyze the tumor tissue to get information of a hereditary nature?

Furthermore, current NCCN BRCA1/2 criteria testing guidelines do not adequately account for the full range of clinical presentations described to date as associated with BRCA1/2 pathogenic/likely pathogenic variant. Like pancreatic cancer, CCA should also be considered an uncommon tumor of the HBOC spectrum.

In the future, not too far away, it will be appropriate that all CCA's patients should undergo genetic testing for BRCA1/2 mutations to identify carriers of pathogenic variants. This is crucial as a referral to genetic screening for the relatives of affected individuals carrying germline BRCA1/2 alterations.

\section{Fig. 1 - Genealogical tree of the family with HBOC syndrome.}

LEGEND: CCA = cholangiocarcinoma; wt = wild type; $y y=$ years.

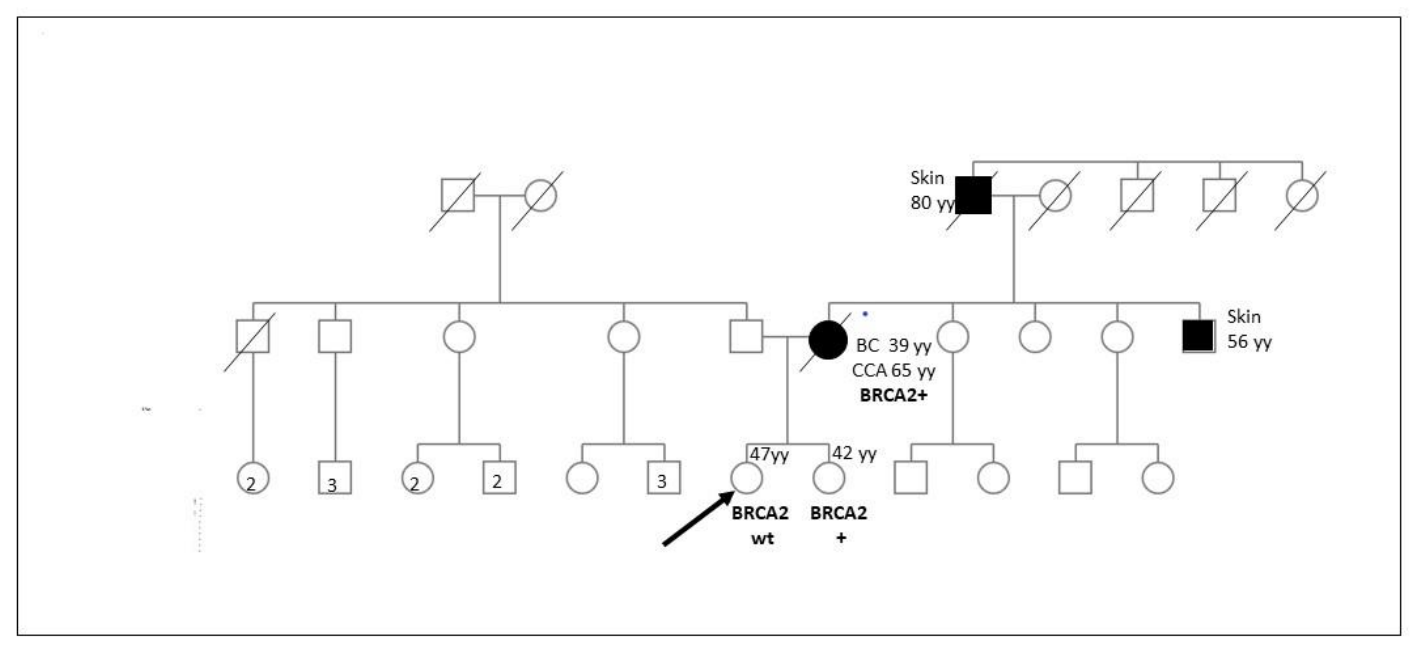

\section{Conflict of interests}

The Authors declare the absence of conflicts of interests. 


\section{Reference}

1. Digennaro, M.; Sambiasi, D.; Tommasi, S.; Pilato, B.; Diotaiuti, S.; Kardhashi, A.; Trojano, G.; Tufaro, A.; Paradiso, A.V. Hereditary and non-hereditarybranches of familyeligible for BRCA test: cancers in other sites. Hered Cancer Clin Pract 2017, 15, 7.

2. Paradiso, A.V.; Digennaro, M.; Patruno, M.; De Summa, S.; Tommasi, S.; Berindan-Neagoe, I. BRCA germline mutation test for all woman with ovariancancer? BMC Cancer 2019, 9, 641.

3. Breast Cancer Linkage Consortium. Cancer risks in BRCA2 mutation carriers. J Natl Cancer Inst 1999, 91, 1310-6.

4. Van Asperen, C.J.; Brohet, R.M.; Meijers-Heijboer, E.J.; Hoogerbrugge, N.; Verhoef, S.; Vasen, H.F.; Ausems, M.G.; Menko, F.H.; Gomez Garcia, E.B.; Klijn, J.G. Cancer risks in BRCA2 families: estimates for sites other than breast and ovary. J Med Genet 2005, 42, 711-9.

5. NCCN Guidelines Version 3.2019 Hereditary Breast and/or Ovarian Cancer Syndrome-Testing Criteria http://www.nccn.org/professionals/physician_gls/pdf/genetics_screening.pdf. Accessed on web 10/30/19.

6. Golan, T.; Raitses-Gurevich, M.; Kelley, R.K.; Bocobo, A.G.; Borgida, A.; Shroff, R.T.; Holter, S.; Gallinger, S.; Ahn, D.H.; Aderka, D. et al. Overall survival and clinical characteristics of BRCAassociated cholangiocarcinoma: a multicenter retrospective study. Oncologist 2017, 22, 804-810.

7. Churi, C.R.; Shroff, R.; Wang, Y.; Rashid, A.; Kang, H.C.; Weatherly, J.; Zuo, M.; Zinner, R.; Hong, D.; Meric-Bernstam, F.; et al. Mutation profiling in cholangiocarcinoma: prognostic and therapeutic implications. PLOS ONE 2014, 9, e115383.

8. Sambiasi, D.; Lambo, R.; Pilato, B.; Tommasi, S.; Trojano, G.; Kardhashi, A.; Digennaro, M.; Trojano, V.; Simone, G.; Paradiso, A. BRCA1/2 and clinicaloutcome in a monoinstitutionalcohort of women with hereditarybreastcancer. Oncol Rep 2014, 31, 365-9.

9. Benafif, S.; Hall, M. An update on PARP inhibitors for the treatment of cancer. Onco Targets Ther 2015, 8, 519-8.

10. Nakamura, H.; Arai, Y.; Totoki, Y.; Shirota, T.; Elzawahry, A.; Kato, M.; Hama, N.; Hosoda, F.; Urushidate, T.; Ohashi S et al. Genomic spectra of biliary tract cancer. Nat Genet 2015, 47, 1003-10.

11. Brandi, G.; Venturi, M.; Pantaleo, M.A.; Ercolani. G.; GICO. Cholangiocarcinoma: Current opinion on clinical practice diagnostic and therapeutic algorithms: A review of the literature and a longstanding experience of a referral center. Dig Liver Dis 2016, 48, 231-41.

12. Gourley, C.; Balmaña, J.; Ledermann, J.; Serra, V.; Dent, R.; Loibl, S.; Pujade-Lauraine, E.; Boulton, S.J. Moving From Poly (ADP-Ribose) Polymerase Inhibition to Targeting DNA Repair and DNA Damage Response in Cancer Therapy. J Clin Oncol 2019, 37, 2257-69. 